International Journal of Engineering \& Technology, $7(4.10)(2018) 573-576$
International Journal of Engineering \& Technology
SPC

\title{
Vertex Hyper-Zagreb Index of Graphs
}

\author{
K.Pattabiraman and M.Seenivasan \\ Department of Mathematics \\ Annamalai University \\ Annamalai Nagar-608002 \\ Tamil Nadu \\ India \\ *Corresponding author E-mail: emseeni@ rediffmail.com
}

\begin{abstract}
In this paper, we obtain the vertex hyper-Zagreb index for some composite graphs. Using the results obtained here, the vertex hyperZagreb index for some important classes of graphs are obtained.
\end{abstract}

Keywords: Composite graphs; F-index; Hyper-Zagreb index; Topological index ; Zagreb index; .

\section{Introduction}

A chemical graph is a graph whose vertices denote atoms and edges denote bonds between those atoms of any underlying chemical structure. A topological index for a (chemical) graph $\mathrm{G}$ is a numerical quantity invariant under automorphisms of $\mathrm{G}$ and it does not depend on the labeling or pictorial representation of the graph. Topological indices and graph invariants based on the distances between vertices of a graph or vertex degrees are widely used for characterizing molecular graphs, establishing relationships between structure and properties of molecules, predicting biological activity of chemical compounds, and making their chemical applications. These indices may be used to derive quantitative structure-property or structure activity relationships (QSPR/QSAR) for more details see [ Bollobs and Erds [3], Gutman and Trinajstic[13], Randic [17], Todeschini and Consonni [20]].

For a (molecular) graph $\mathrm{G}$, The first Zagreb index $\mathrm{M}_{1}(\mathrm{G})$ is the equal to the sum of the squares of the degrees of the vertices, and the second Zagreb index $\mathrm{M}_{2}(\mathrm{G})$ is the equal to the sum of the products of the degrees of pairs of adjacent vertices, that is,

$$
\begin{aligned}
\mathrm{M}_{1}(\mathrm{G}) & =\sum_{u \in V(G)} d_{G}{ }^{2}(u) \\
& =\sum_{u v \in E(G)}\left(d_{G}(u)+d_{G}(v)\right) \\
\mathrm{M}_{2}(\mathrm{G}) & =\sum_{u v \in E(G)} d_{G}(u) d_{G}(v) .
\end{aligned}
$$

There are different types of Zagreb indices were studied by various authors. One of the important version of Zagreb indices, the vertex first and second Zagreb indices were proposed by Tavakoli et al.[10]. They are defined as

$$
\bar{M}^{*}(\mathrm{G})=\sum_{\{u, v\} \subseteq V(G)}\left(d_{G}(u)+d_{G}(v)\right),
$$

$$
\bar{M}_{2}^{*}(\mathrm{G})=\sum_{\{u, v\} \subseteq V(G)} d_{G}(u) d_{G}(v) .
$$

Shirdel et al. [9] proposed the another version of Zagreb index, namely, hyper- Zagreb index, which is defined as

$$
\mathrm{HM}(\mathrm{G})=\sum_{u v \in E(G)}\left(d_{G}(u)+d_{G}(v)\right)^{2} .
$$

Similarly, the hyper-Zagreb co index is defined as

$$
\overline{H M}(\mathrm{G})=\sum_{u v \notin E(G)}\left(d_{G}(u)+d_{G}(v)\right)^{2} .
$$

In this connection, we introduce the new version of $\mathrm{Za}$ greb index called vertex hyper-Zagreb index which is defined as

$$
\overline{H M}_{1}^{*}(G)=\sum_{\{u v\} \subseteq E(G)}\left(d_{G}(u)+d_{G}(v)\right)^{2} .
$$

The $F$-index was introduced by Furtula and Gutman [4], and it is defined as

$$
\begin{aligned}
\mathrm{F}=\mathrm{F}(\mathrm{G}) & =\sum_{u \in V(G)} d_{G}{ }^{3}(u) \\
& =\sum_{u v E(G)}\left(d_{G}{ }^{2}(u)+{d_{G}}^{2}(u)\right)
\end{aligned}
$$

They established a few basic properties of the forgotten topological index and show that it can significantly enhance the physicchemical applicability of the first Zagreb index.

Khalifeh et al. [6] obtained the first and second Zagreb indices of the Cartesian, join, composition, disjunction and symmetric difference of two graphs. Ashrafi et al. [1] obtained the first and second Zagreb coindices of the Cartesian, join, composition, disjunction and symmetric difference of two graphs.

In Fath-Tabar [3] and shirdel et al. [9] the hyper and third Zagreb indices of some graph operations are obtained. The mathematical properties for the third and hyper-Zagreb coindices of graph operations containing the Cartesian product and composition are explained by Gao et al. [5]. Pattabiraman and Seenivasan [7] analyzed Bounds of vertex Zegreb indices of graphs for more details see[ Gutman [8], Gutman [9], ] Gutman ,Das [10], ] Gutman, Furtula, 
Kovijanic Vukicevic and popivoda [11], Gutman, Tomovice , [12]]. In this paper, we obtain the vertex hyper-Zagreb index for some composite graphs. Using the results obtained here, the vertex hyper-Zagreb index for some important classes of graphs are obtained.

\section{Composite Graphs}

Some chemically interesting graphs can be obtained by different graph operations on general or particular graphs. Thus graph operations played a important role in chemical graph theory Doslic [3]. In this view, here we obtain the vertex hyper-Zagreb indices of different types of operations of graphs.

\subsection{Join}

The join $G+H$ of graphs $G$ and $H$ is obtained from the disjoint union of the graphs $G$ and $H$, where each vertex of $G$ is adjacent to each vertex of $H$.

\section{Theorem 1.}

Let $\mathrm{G}_{\mathrm{i}}$ be a graph with $\mathrm{n}_{\mathrm{i}}$ vertex and $\mathrm{m}_{\mathrm{i}}$ edges, $\mathrm{i}=1,2$. Then $\overline{H M}^{*}\left(\mathrm{G}_{1}+\mathrm{G}_{2}\right)=\overline{H M}^{*}\left(\mathrm{G}_{1}\right)+4 \mathrm{n}_{2} \bar{M}^{*}\left(\mathrm{G}_{1}\right)+\overline{H M}^{*}\left(\mathrm{G}_{2}\right)+$ $4 \mathrm{n}_{1} \bar{M}_{1}^{*}\left(\mathrm{G}_{2}\right)+\mathrm{n}_{2} \bar{M}_{1}\left(\mathrm{G}_{1}\right)+\mathrm{n}_{1} \mathrm{M}_{1}\left(\mathrm{G}_{2}\right)$

$+\mathrm{n}_{1} \mathrm{n}_{2}\left(4 \mathrm{n}_{1} \mathrm{n}_{2}-2 \mathrm{n}_{1}-2 \mathrm{n}_{2}+\left(\mathrm{n}_{1}+\mathrm{n}_{2}\right)^{2}\right)$ $+4\left(n_{1}+n_{2}\right)\left(m_{1} n_{2}+m_{2} n_{1}\right)+8 m_{1} m_{2}$.

Proof of theorem 1. Set $V\left(G_{1}\right)=\left\{u_{1}, u_{2}, \ldots, u_{n}\right\}$ and

$V\left(G_{2}\right)=\left\{v_{1}, v_{2}, \ldots, v_{m}\right\}$.

By definition of the join of two graphs, one can see that,

$\mathrm{d}_{\mathrm{G} 1+\mathrm{G} 2}(\mathrm{x})= \begin{cases}d G_{1}(X)+n_{2} ; & \text { if } x \in V\left(G_{1}\right) \\ d G_{2}(X)+n_{1} ; & \text { if } x \in V\left(G_{2}\right)\end{cases}$

By the definition of vertex hyper Zagreb index,

$$
\begin{gathered}
\overline{H M}_{1}^{*}\left(\mathrm{G}_{1}+\mathrm{G}_{2}\right)=\sum_{\{u, v\} \subseteq V\left(G_{1}+G_{2}\right)}\left(\mathrm{d}_{\mathrm{G}_{1}+G_{2}}(\mathrm{u})+\mathrm{d}_{\mathrm{G}_{1}+G_{2}}(\mathrm{v})\right)^{2} \\
=\sum_{\{u, v\} \subseteq V\left(G_{1}\right)}\left(\mathrm{d}_{\mathrm{G}_{1}+G_{2}}(\mathrm{u})+\mathrm{d}_{\mathrm{G}_{1}+G_{2}}(\mathrm{v})\right)^{2} \\
+\sum_{\{u, v\} \subseteq V\left(G_{2}\right)}\left(\mathrm{d}_{\mathrm{G}_{1}+G_{2}}(\mathrm{u})+\mathrm{d}_{\mathrm{G}_{1}+G_{2}}(\mathrm{v})\right)^{2} \\
+\sum_{u \in V\left(G_{1}\right)} \sum_{\mathrm{v} \in \mathrm{v}\left(\mathrm{G}_{2}\right)}\left(\mathrm{d}_{\mathrm{G}_{1}+G_{2}}(\mathrm{u})+\mathrm{d}_{\mathrm{G}_{1}+G_{2}}(\mathrm{v})\right)^{2} \\
=\sum_{\{u, v\} \subseteq V\left(G_{1}\right)}\left(\mathrm{d}_{\mathrm{G}_{1}}(\mathrm{u})+\mathrm{d}_{\mathrm{G}_{1}}(\mathrm{v})+2 \mathrm{n}_{2}\right)^{2} \\
+\sum_{\{u, v\} \subseteq V\left(G_{2}\right)}\left(\mathrm{d}_{\mathrm{G}_{2}}(\mathrm{u})+\mathrm{d}_{\mathrm{G}_{2}}(\mathrm{v})+2 \mathrm{n}_{1}\right)^{2} \\
+\sum_{u \in V\left(G_{1}\right)} \sum_{\mathrm{v} \in \mathrm{v}\left(\mathrm{G}_{2}\right)}\left(\mathrm{d}_{\mathrm{G}_{1}}(\mathrm{u})+\mathrm{n}_{2}+\mathrm{d}_{\mathrm{G}_{2}}(\mathrm{v})+\mathrm{n}_{1}\right)^{2} \\
=\sum_{\{u, v\} \subseteq V\left(G_{1}\right)}\left(\left(\mathrm{d}_{\mathrm{G}_{1}}(\mathrm{u})+\mathrm{d}_{\mathrm{G}_{1}}(\mathrm{v})\right)^{2}+4 n_{2}{ }^{2}+4 n_{2}\left(d_{G_{1}}(u)+d_{G_{1}}(v)\right)\right. \\
+\sum_{\{u, v\} \subseteq V\left(G_{2}\right)}\left(\left(\mathrm{d}_{\mathrm{G}_{2}}(\mathrm{u})+\mathrm{d}_{\mathrm{G}_{2}}(\mathrm{v})\right)^{2}+4 n_{1}{ }^{2}+4 n_{1}\left(d_{G_{2}}(u)+d_{G_{2}}(v)\right)\right. \\
+\sum_{u \in V\left(G_{1}\right)} \sum_{\mathrm{v} \in \mathrm{v}\left(\mathrm{G}_{2}\right)}\left(\mathrm{d}_{\mathrm{G}_{1}}^{2}(\mathrm{u})+\mathrm{d}^{2} \mathrm{G}_{\mathrm{G}_{2}}(\mathrm{v})+\left(\mathrm{n}_{1}+\mathrm{n}_{2}\right)^{2}+2 d_{G_{1}}(u)\left(\mathrm{n}_{1}+\mathrm{n}_{2}\right)\right. \\
\left.+2 d_{G_{2}}(v)\left(\mathrm{n}_{1}+\mathrm{n}_{2}\right)+2 d_{G_{1}}(u) d_{G 2}(v)\right) . \\
=\mathrm{HM}_{1}^{*}\left(\mathrm{G}_{1}\right)+4 \mathrm{n}_{2}^{2} \frac{n_{1}\left(n_{1}-1\right)}{2}+4 \mathrm{n}_{2} \bar{M}_{1}^{*}\left(\mathrm{G}_{1}\right)
\end{gathered}
$$

$$
\begin{aligned}
& +\mathrm{HM}^{*}{ }_{1}\left(\mathrm{G}_{2}\right)+4 \mathrm{n}^{2}{ }_{1} \frac{n_{2}\left(n_{2}-1\right)}{2}+4 \mathrm{n}_{1} \bar{M}_{1}^{*}\left(\mathrm{G}_{2}\right) \\
& +\mathrm{n}_{2} \mathrm{M}_{1}\left(\mathrm{G}_{1}\right)+\mathrm{n}_{1} \mathrm{M}_{1}\left(\mathrm{G}_{2}\right)+\left(\mathrm{n}_{1}+\mathrm{n}_{2}\right)^{2} \mathrm{n}_{1} \mathrm{n}_{2} \\
& +4 \mathrm{~m}_{1} \mathrm{n}_{2}\left(\mathrm{n}_{1}+\mathrm{n}_{2}\right)+4 \mathrm{~m}_{2}\left(\mathrm{n}_{1}+2\right) \mathrm{n}_{1}+8 \mathrm{~m}_{1} \mathrm{~m}_{2}
\end{aligned}
$$

Hence

$$
\begin{aligned}
\overline{H M}_{1}^{*}\left(G_{1}+G_{2}\right) & =\overline{H M}_{1}^{*}\left(\mathrm{G}_{1}\right)+4 \mathrm{n}_{2} \bar{M}_{1}^{*}\left(\mathrm{G}_{1}\right) \\
& +\overline{H M}^{*}\left(\mathrm{G}_{2}\right)+4 \mathrm{n}_{1} \bar{M}_{1}^{*}\left(\mathrm{G}_{2}\right) \\
& +\mathrm{n}_{2} \mathrm{M}_{1}\left(\mathrm{G}_{1}\right)+\mathrm{n}_{1} \mathrm{M}_{1}\left(\mathrm{G}_{2}\right) \\
& +\mathrm{n}_{2}\left(4 \mathrm{n}_{1} \mathrm{n}_{2}-\mathrm{n}_{1}-2 \mathrm{n}_{2}+\left(\mathrm{n}_{1}+\mathrm{n}_{2}\right)^{2}\right) \\
& +4\left(\mathrm{n}_{1}+\mathrm{n}_{2}\right)\left(\mathrm{m}_{1} \mathrm{n}_{2}+\mathrm{m}_{2} \mathrm{n}_{1}\right) \\
& +8 \mathrm{~m}_{1} \mathrm{~m}_{2} .
\end{aligned}
$$

Example 2.1. The complete bipartite graph $\mathrm{K}_{\mathrm{p}, \mathrm{q}}$ can be defined as $\mathrm{K}_{\mathrm{p}, \mathrm{q}}=\overline{K_{p}}+\overline{K_{q}}$. The vertex hyper-Zagreb index of $\mathrm{K}_{\mathrm{p}, \mathrm{q}}$ is $\mathrm{pq}\left(4 \mathrm{pq}-2 \mathrm{p}-2 \mathrm{q}+(\mathrm{p}+\mathrm{q})^{2}\right)$.

Example 2.2. Let $\mathrm{G}$ be a graph with $\mathrm{n}$ vertices and $\mathrm{m}$ edges. Then

$$
\begin{aligned}
\overline{H M}^{*}\left(\mathrm{G}+\mathrm{K}_{\mathrm{p}}\right) & =\overline{H M}_{1}^{*}(\mathrm{G})+4 \mathrm{p} \bar{M}^{*}(\mathrm{G})+\mathrm{pM}_{1}(\mathrm{G}) \\
& +\mathrm{p}(\mathrm{p}-1)^{2}(2 \mathrm{p}+5 \mathrm{n}-2) \\
& \left.+\mathrm{np}(4 \mathrm{np}-2)(\mathrm{n}+\mathrm{p})+(\mathrm{n}+\mathrm{p})^{2}\right) \\
& +2(\mathrm{n}+\mathrm{p})(2 \mathrm{mp}+\mathrm{np}(\mathrm{p}-1)) \\
& +4 \mathrm{mp}(\mathrm{p}-1)
\end{aligned}
$$

Using Example 2.2, we obtain the following examples.

Example 2.3. The Suspension of $G$ is defined as join of $G$ with a single vertex. The vertex hyper-Zagreb index of $\mathrm{G}+\mathrm{K}_{1}$ is $\overline{H M}_{1}^{*}(\mathrm{G})$ $+4 \bar{M}_{1}^{*}(\mathrm{G})+\mathrm{M}_{1}(\mathrm{G})+\mathrm{n}\left(\mathrm{n}^{2}+8 \mathrm{n}-1\right)+4 \mathrm{mp}(\mathrm{p}-1)$.

Example 2.4. The fan and wheel graphs are defined as $\mathrm{P}_{\mathrm{n}}+\mathrm{K}_{1}$ and $\mathrm{C}_{\mathrm{n}}+\mathrm{K}_{1}$. The vertex hyper-Zagreb indices of these graphs are

(i) $\overline{H M}^{*}\left(C_{n}+K_{1}\right)=n\left(n^{2}+24 n-13\right)+4 m(n+1)$.

(ii) $\overline{H M}_{1}^{*}\left(P_{n}+K_{1}\right)=n\left(n^{2}+36 n-71\right)+4 m(n+1)+42$.

Example 2.5. The Cone graph $C_{n, p}$ is defined as $C_{n}+K_{p}$. So the vertex hyper Zagreb index of cone graph is given

$$
\begin{aligned}
\overline{H M}^{*}\left(C_{n}+K_{p}\right) & =8 \mathrm{n}(\mathrm{n}-1)(\mathrm{p}+1)+4 \mathrm{pn} \\
& +\mathrm{p}(\mathrm{p}-1)^{2}(2 \mathrm{p}+5 \mathrm{n}-2) \\
& +\mathrm{np}\left(4 \mathrm{np}-2(\mathrm{n}+\mathrm{p})+(\mathrm{n}+\mathrm{p})^{2}\right) \\
& +2(\mathrm{n}+\mathrm{p}) \mathrm{np}(\mathrm{p}+1))+4 \mathrm{np}(\mathrm{p}-1) .
\end{aligned}
$$

\subsection{Union}

Let $\mathrm{G}$ and $\mathrm{H}$ be two connected graphs with $\mathrm{V}(\mathrm{G})$ and

$\mathrm{V}(\mathrm{H})$ vertices, respectively. The union of $\mathrm{G}$ and $\mathrm{H}$ is the graph denoted by $\mathrm{G} U \mathrm{H}$ with the vertex set $\mathrm{V}(\mathrm{G}) \mathrm{U} \mathrm{V}(\mathrm{H})$ and the edge set $E(G) \cup E(H)$, where $V(G)$ and $V(H)$ are disjoint. The degree of a vertex $u$ in $\mathrm{G} U \mathrm{H}$ is degree of that vertex in the components $\mathrm{G}$ and $\mathrm{H}$ that contains it. Now we obtain the vertex hyper-zagreb index of the union of two graphs.

Theorem 2. Let $\overline{H M}_{1}^{*}\left(\mathrm{G}_{1} \cup_{\mathrm{G}_{2}}\right)=\overline{H M}_{1}^{*}\left(\mathrm{G}_{1}\right)+\overline{H M}_{1}^{*}\left(\mathrm{G}_{2}\right)+$ $\mathrm{n}_{2} \mathrm{M}_{1}\left(\mathrm{G}_{1}\right)+\mathrm{n}_{1} \mathrm{M}_{1}\left(\mathrm{G}_{2}\right)+4 \mathrm{~m}_{1} \mathrm{~m}_{2}$.

Proof of theorem 2. From the structure of the GUH, we have

$$
\begin{aligned}
\overline{H M}^{*}\left(\mathrm{G}_{1} \cup \mathrm{G}_{2}\right) & =\sum_{\{u, v\} \in V(G)}\left(\mathrm{d}_{\mathrm{G}}(\mathrm{u})+\mathrm{d}_{\mathrm{G}}(\mathrm{v})\right)^{2} \\
= & \sum_{\{u, v\} \in V\left(G_{1}\right)}\left(\mathrm{d}_{\mathrm{G}_{1}}(\mathrm{u})+\mathrm{d}_{\mathrm{G}_{1}}(\mathrm{v})\right)^{2} \\
& +\sum_{\{u, v\} \in V\left(\mathrm{G}_{2}\right)}\left(\mathrm{d}_{\mathrm{G}_{2}}(\mathrm{u})+\mathrm{d}_{\mathrm{G}_{2}}(\mathrm{v})\right)^{2}
\end{aligned}
$$




$$
\begin{aligned}
& +\sum_{u \in V\left(G_{1}\right)} \sum_{v \in V\left(G_{2}\right)}\left(d_{G_{1}}(u)+d_{G_{2}}(v)\right)^{2} \\
= & \overline{H M}_{1}^{*}\left(\mathrm{G}_{1}\right)+\overline{H M}^{*}\left(\mathrm{G}_{2}\right)+\mathrm{n}_{2} \mathrm{M}_{1}\left(\mathrm{G}_{1}\right)+\mathrm{n}_{1} \mathrm{M}_{1}\left(\mathrm{G}_{2}\right)+4 \mathrm{~m}_{1} \mathrm{~m}_{2} .
\end{aligned}
$$

\subsection{Cartesian Product}

The Cartesian product, $\mathrm{G} \square \mathrm{H}$, of the graphs $\mathrm{G}$ and $\mathrm{H}$ has the vertex set $\mathrm{V}(\mathrm{G} \square \mathrm{H})=\mathrm{V}(\mathrm{G}) \times \mathrm{V}(\mathrm{H})$ and $(\mathrm{u}, \mathrm{x})(\mathrm{v}, \mathrm{y})$ is an edge of $\mathrm{G}$ $\square \mathrm{H}$ if $\mathrm{u}=\mathrm{v}$ and $\mathrm{xy} \in \mathrm{E}(\mathrm{H})$ or, $\mathrm{uv} \in \mathrm{E}(\mathrm{G})$ and $\mathrm{x}=\mathrm{y}$. To each vertex $u \in V(G)$, there is an isomorphic copy of $H$ in $G \square \mathrm{H}$ and to each vertex $v \in V(H)$, there is an isomorphic copy of $G$ in $G \square H$.

Theorem 3. Let $\mathrm{G}_{\mathrm{i}}$ be a graph with $\mathrm{n}_{\mathrm{i}}$ vertex and $\mathrm{m}_{\mathrm{i}}$ edges, $\mathrm{i}=1,2$. Then

$$
\begin{aligned}
\overline{H M}\left(\mathrm{G}_{1} \square \mathrm{G}_{2}\right)= & \frac{M_{1}\left(G_{1}\right)}{2}\left[2 \mathrm{n}^{2} \mathrm{n}_{1}-3 \mathrm{n}_{2}-18 \mathrm{~m}_{2}\right] \\
& +\frac{M_{1}\left(G_{2}\right)}{2}\left[2 \mathrm{n}^{2}{ }_{1} \mathrm{n}_{2}-3 \mathrm{n}_{1}-18 \mathrm{~m}_{1}\right] \\
& -2 \mathrm{n}_{1} \mathrm{~m}_{2}\left(\mathrm{G}_{2}\right)-2 \mathrm{n}_{2} \mathrm{~m}_{2}\left(\mathrm{G}_{1}\right) \\
& -\mathrm{n}_{2} \mathrm{~F}\left(\mathrm{G}_{1}\right)-\mathrm{n}_{1} \mathrm{~F}\left(\mathrm{G}_{2}\right) \\
& +4\left(\mathrm{n}_{1} \mathrm{~m}_{2}+\mathrm{n}_{2} \mathrm{~m}_{1}\right)^{2}+8 \mathrm{~m}_{1} \mathrm{~m}_{2}\left(\mathrm{n}_{1} \mathrm{n}_{2}-2\right)
\end{aligned}
$$

The proof of the following theorem follows by applying Theorem 4.1 of [5] and Theorem 3 respectively, and using the fact that $\mathrm{HM}(\mathrm{G})=\overline{H M}^{*}(\mathrm{G})-\overline{H M}(\mathrm{G})$.

Theorem 4 . Let $\mathrm{G}_{\mathrm{i}}$ be a graph with $\mathrm{n}_{\mathrm{i}}$ vertex and $\mathrm{m}_{\mathrm{i}}$ edges, $\mathrm{i}=1$, 2. Then $\overline{H M}^{*}\left(\mathrm{G}_{1} \square \mathrm{G}_{2}\right)=\mathrm{n}_{1} \mathrm{HM}\left(\mathrm{G}_{2}\right)+\mathrm{n}_{2} \mathrm{HM}\left(\mathrm{G}_{1}\right)+$ $\frac{M_{1}\left(G_{1}\right)}{2}\left[2 \mathrm{n}^{2}{ }_{2} \mathrm{n}_{1}-3 \mathrm{n}_{2}+6 \mathrm{~m}_{2}\right]+\frac{M_{1}\left(G_{2}\right)}{2}\left[2 \mathrm{n}^{2}{ }_{1} \mathrm{n}_{2}-3 \mathrm{n}_{1}+6 \mathrm{~m}_{1}\right]-2\left[\mathrm{n}_{1}\right.$ $\left.\mathrm{M}_{2}\left(\mathrm{G}_{2}\right)+\mathrm{n}_{2} \mathrm{M}_{2}\left(\mathrm{G}_{1}\right)\right]-\left[\mathrm{n}_{2} \mathrm{~F}\left(\mathrm{G}_{1}\right)+\mathrm{n}_{1} \mathrm{~F}\left(\mathrm{G}_{2}\right)\right]$

$+4\left(\mathrm{n}_{1} \mathrm{~m}_{2}+\mathrm{n}_{2} \mathrm{~m}_{1}\right)^{2}+8 \mathrm{~m}_{1} \mathrm{~m}_{2}\left(\mathrm{n}_{1} \mathrm{n}_{2}-2\right)$.

By using Theorem 4 , we obtain the following examples.

Example 2.6. The $\mathrm{C}_{4}$-nanotorus denoted by $\mathrm{TC}_{4}(\mathrm{~m}, \mathrm{n})$, is defined as the Cartesian product of two cycles.

Then $\overline{H M}_{1}^{*}\left(\mathrm{C}_{\mathrm{n}} \square \mathrm{C}_{\mathrm{m}}\right)=4 \mathrm{~nm}(8 \mathrm{~nm}+1)$.

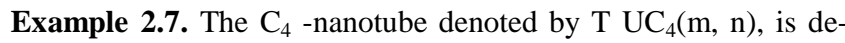
fined as the Cartesian product of two paths. Then

$$
{ }_{H M}{ }^{*}\left(\mathrm{P}_{\mathrm{n}} \square \mathrm{P}_{\mathrm{m}}\right)=4 \mathrm{~m}^{2}\left[6\left(\mathrm{n}^{2}-\mathrm{n}\right)+1\right]-12 \mathrm{~m}\left(\mathrm{n}^{2}-\mathrm{n}\right)-2\left(\mathrm{n}^{2}-32 \mathrm{n}+5\right) \text {. }
$$

Example 2.8. The vertex hyper-Zagreb index of the Cartesian product of a path and a cycle is $\overline{H M}_{1}^{*}\left(\mathrm{P}_{\mathrm{n}} \square \mathrm{C}_{\mathrm{m}}\right)=\mathrm{n}^{2}\left(12 \mathrm{~m}^{2}-9 \mathrm{~m}+\right.$ $4)+16 m n-21 m+4 n-4 m^{2}(6 n-1)+2$.

Example 2.9. The Ladder graph $\mathrm{L}_{\mathrm{m}}$ made by $\mathrm{m}$ square is the Cartesian product of $\mathrm{P}_{2}$ and $\mathrm{P}_{\mathrm{m}}$. The vertex hyper-Zagreb index of $\mathrm{L}_{n}$ is given by $\overline{H M}_{1}^{*}\left(\mathrm{~L}_{\mathrm{n}}\right)=70 \mathrm{n}^{2}-8 \mathrm{n}+6$.

\subsection{Double Graph}

Let $G$ be a graph with $V(G)=\left\{v_{1}, v_{2}, \ldots, v_{n}\right\}$. The vertices of the double graph $G^{*}$ are given by the two sets $X=\left\{x_{1}, x_{2}, \ldots, x_{n}\right\}$ and $\mathrm{Y}=\left\{\mathrm{y}_{1}, \mathrm{y}_{2}, \ldots, \mathrm{y}_{\mathrm{n}}\right\}$. Thus, for each vertex $\mathrm{v}_{\mathrm{i}} \in \mathrm{V}(\mathrm{G})$, there are two vertices $x_{i}$ and $y_{i}$ in $V\left(G^{*}\right)$. The double graph $G^{*}$ includes the initial edge set of each copy of $G$, and for any edge $v_{i} v_{j} \in E(G)$, two more edges $x_{i} y_{j}$ and $x_{j} y_{i}$ are added.

Theorem 5. Let $\mathrm{G}$ be a connected graph. Then $\overline{H M}_{1}^{*}\left(\mathrm{G}^{*}\right)=$ $16 \mathrm{HM}(\mathrm{G})+16 \overline{H M}(\mathrm{G})+16 \mathrm{M}_{1}(\mathrm{G})$.

The proof of the above theorem follows by applying Theorems 2.3 and 2.4 of [8], respectively.

\subsection{Corona Product}

For given graphs $\mathrm{G}$ and $\mathrm{H}$, we define their corona product $\mathrm{G} \circ \mathrm{H}$ as the graph obtained by taking $|\mathrm{V}(\mathrm{G})|$ copies of $\mathrm{H}$ and joining each vertex of the $i$-th copy with vertex $v_{i} \in V(G)$. Obviously, $\mid V$ $(\mathrm{G} \circ \mathrm{H})|=| \mathrm{V}(\mathrm{G}) \mid(1+|\mathrm{V}(\mathrm{H})|)$ and $|\mathrm{E}(\mathrm{G} \circ \mathrm{H})|=|\mathrm{E}(\mathrm{G})|+|\mathrm{V}(\mathrm{G})|$ $(|\mathrm{V}(\mathrm{H})|+|\mathrm{E}(\mathrm{H})|)$. From the structure of the corona product of two graphs, we have the degree of a vertex $\mathrm{x}$ in $\mathrm{V}\left(\mathrm{G}_{1} \circ \mathrm{G}_{2}\right)$ is given by

$\mathrm{d}_{\mathrm{G} 1 \circ \mathrm{G} 2}(\mathrm{x})=\left\{\begin{array}{cc}d G_{1}(x)+\left|V\left(G_{2}\right)\right|, & \text { if } x \in V\left(G_{1}\right) \\ d G_{2}(x)+1, & \text { if } x \in V\left(G_{2}, i\right), i=1,2, \ldots,\left|V\left(G_{1}\right)\right|\end{array}\right.$

where $G_{2, i}$ is the $i^{\text {th }}$ copy of $G_{2}$

The following theorem is presented in [11].

Theorem 6. Let $\mathrm{G}_{\mathrm{i}}$ be a graph with $\mathrm{n}_{\mathrm{i}}$ vertex and $\mathrm{m}_{\mathrm{i}}$ edges, $\mathrm{i}=1,2$. Then

$$
\begin{aligned}
& \overline{H M}\left(\mathrm{G}_{1} \circ \mathrm{G}_{2}\right)=2\left[\overline{M_{2}}\left(\mathrm{G}_{1}\right)+\mathrm{n}_{2} \overline{M_{1}}\left(\mathrm{G}_{1}\right)+\mathrm{n}_{1} \overline{M_{1}}\left(\mathrm{G}_{2}\right)\right. \\
& \left.+\overline{M_{2}}\left(\mathrm{G}_{2}\right) \mathrm{n}_{1}\right]-\mathrm{F}\left(\mathrm{G}_{1}\right)-\mathrm{n}_{1} \mathrm{~F}\left(\mathrm{G}_{2}\right) \\
& +\left(\mathrm{n}_{1}+\mathrm{n}_{1} \mathrm{n}_{2}-3 \mathrm{n}_{2}-1\right) \mathrm{M}_{1}\left(\mathrm{G}_{1}\right) \\
& +\mathrm{n}_{1}\left(\mathrm{n}_{1}+\mathrm{n}_{1} \mathrm{n}_{2}-4\right) \mathrm{M}_{1}\left(\mathrm{G}_{2}\right) \\
& +\mathrm{n}_{2}^{2}\left(\mathrm{n}_{1}\left(\mathrm{n}_{1}-1\right)-2 \mathrm{~m}_{1}\right) \\
& +\mathrm{n}_{1}\left(\mathrm{n}_{2}\left(\mathrm{n}_{2}-1\right)-2 \mathrm{~m}_{2}\right) \\
& +\mathrm{n}_{1} \mathrm{n}_{2}\left(8 \mathrm{n}_{1} \mathrm{~m}_{2}+3 \mathrm{n}_{1} \mathrm{n}_{2}-8 \mathrm{~m}_{2}-3 \mathrm{n}_{2}\right) \\
& +2\left(\mathrm{n}_{1}-1\right)\left(4 \mathrm{~m}_{1} \mathrm{~m}_{2}+2 \mathrm{n}_{2} \mathrm{~m}_{1}+2 \mathrm{~m}_{2}^{2} \mathrm{n}_{1}\right) \\
& +\left(\mathrm{n}_{1}\left(\mathrm{n}_{2}+1\right)-1\right)\left[\mathrm{n}_{1} \mathrm{n}^{2}{ }_{2}+4 \mathrm{n}_{2} \mathrm{~m}_{1}+\mathrm{n}_{1} \mathrm{n}_{2}+4 \mathrm{n}_{1} \mathrm{~m}_{2}\right] \\
& \left(6 \mathrm{n}^{2} \mathrm{~m}_{1}+6 \mathrm{n}_{1} \mathrm{~m}_{2}+\mathrm{n}_{1} \mathrm{n}_{2}\left(\mathrm{n}^{2}{ }_{2}+1\right)\right) \text {. } \\
& \overline{H M}_{1}^{*}\left(\mathrm{G}_{1} \circ \mathrm{G}_{2}\right)=\left(\mathrm{n}_{2}+1\right)^{3} \mathrm{HM}\left(\mathrm{G}_{1}\right) \\
& +\mathrm{m}_{2} \mathrm{HM}\left(\mathrm{G}_{2}\right)+\left(\mathrm{m}_{1}+\left(\mathrm{n}_{2}+1\right)^{2} \overline{H M}\left(\mathrm{G}_{1}\right)\right. \\
& +8 \mathrm{~m}_{1} \overline{M_{1}}\left(\mathrm{G}_{2}\right)-\mathrm{n}_{2}\left(\mathrm{n}_{2}+1\right)^{2} \mathrm{~F}\left(\mathrm{G}_{1}\right) \\
& +\left(\mathrm{n}_{2}+1\right)^{2} \mathrm{n}_{1} \mathrm{n}_{2} \mathrm{M}_{1}\left(\mathrm{G}_{1}\right) \\
& +\left(8 \mathrm{~m}_{2}+\mathrm{n}^{2}{ }_{1}-\mathrm{m}_{1}+\mathrm{n}_{2} \mathrm{~m}_{1}\left(\mathrm{~m}_{1}-1\right)\right) \mathrm{M}_{1}\left(\mathrm{G}_{2}\right) \\
& +16 \mathrm{~m}_{2}^{2}+4 \mathrm{~m}_{1}\left(2 \mathrm{~m}_{2}-2 \mathrm{n}^{2}{ }_{2}+5 \mathrm{n}_{2}\right) \\
& +8\left(\mathrm{n}_{2}+1\right) \mathrm{n}_{1} \mathrm{~m}_{1}\left(\mathrm{n}_{2}+\mathrm{m}_{2}\right)-32 \mathrm{~m}_{1} \mathrm{~m}_{2} \\
& +4 \mathrm{n}^{2}{ }_{1}\left(2 \mathrm{~m}_{2}+\mathrm{n}_{2}\right)+\mathrm{m}_{1}\left(\mathrm{~m}_{1}-1\right)\left(4 \mathrm{~m}^{2}{ }_{2}+8 \mathrm{n}^{2}{ }_{2}+16 \mathrm{n}_{2} \mathrm{~m}_{2}\right) \text {. }
\end{aligned}
$$

For a given graph $\mathrm{G}$, its $\mathrm{t}$-fold bristled graph $\operatorname{Brst}(\mathrm{G})$ is obtained by attaching $t$ vertices of degree 1 to each vertex of $G$. This graph can be represented as the corona product of $\mathrm{G}$ and complement of a complete graph on t vertices. The $t$-fold bristled graph of a given graph is also known as its

$\mathrm{t}$-thorny graph.

Example 2.10. The vertex hyper-Zagreb index of the $\mathrm{t}$-fold bristled graph of $\mathrm{C}_{\mathrm{n}}$ is given by $\overline{H M}_{1}^{*}\left(\mathrm{C}_{\mathrm{n}} \circ \overline{K_{t}}\right)=\mathrm{n}^{2} \mathrm{t}^{3}+10 \mathrm{n}^{2} \mathrm{t}^{2}-2 \mathrm{nt}^{2}+$ $17 n^{2} t-10 n t-8 n+8 n^{2}$.

\subsection{Composition}

The composition $\mathrm{G}[\mathrm{H}]$ of graphs $\mathrm{G}$ and $\mathrm{H}$ with disjoint vertex sets $\mathrm{V}(\mathrm{G})$ and $\mathrm{V}(\mathrm{H})$ and edge sets $\mathrm{E}(\mathrm{G})$ and $\mathrm{E}(\mathrm{H})$ is the graph with vertex set $\mathrm{V}(\mathrm{G}) \times \mathrm{V}(\mathrm{H})$ and $\left(\mathrm{u}_{1}, \mathrm{v}_{1}\right)$ is adjacent with $\left(\mathrm{u}_{2}, \mathrm{v}_{2}\right)$ whenever $\left(u_{1}\right.$ is adjacent with $\left.u_{2}\right)$ or $\left(u_{1}=u_{2}\right.$ and $v_{1}$ is adjacent with $\left.v_{2}\right)$. From the structure of $\mathrm{G}[\mathrm{H}]$, the degree of a vertex $(u, v)$ of $\mathrm{G}[\mathrm{H}]$ is given by $\mathrm{d}_{\mathrm{G}[\mathrm{H}]}((\mathrm{u}, \mathrm{v}))=|\mathrm{V}(\mathrm{H})| \mathrm{d}_{\mathrm{G}}(\mathrm{u})+\mathrm{d}_{\mathrm{H}}(\mathrm{v})$. The following theorem is appeared in Veylaki et al [11].

Theorem 8. Let $\mathrm{G}_{\mathrm{i}}$ be a graph with $\mathrm{n}_{\mathrm{i}}$ vertex and $\mathrm{m}_{\mathrm{i}}$ edges, $\mathrm{i}=1,2$. Then $\quad \overline{H M} \quad\left(\mathrm{G}_{1}\left[\mathrm{G}_{2}\right]\right)=\mathrm{n}_{2}^{2} \mathrm{M}_{1}\left(\mathrm{G}_{1}\right)\left(\mathrm{n}_{2}^{2} \mathrm{n} 1-2 \mathrm{n}_{2}-12 \mathrm{~m}_{2}\right)$ $+\mathrm{M}_{1}\left(\mathrm{G}_{2}\right)\left(\mathrm{n}^{2}{ }_{1} \mathrm{n}_{2}-2 \mathrm{n}_{1}-10 \mathrm{n}_{2} \mathrm{~m}_{1}\right)-\mathrm{n}^{4}{ }_{2} \mathrm{M}_{2}\left(\mathrm{G}_{1}\right)$ 
$-\mathrm{n}_{1} \mathrm{M}_{2}\left(\mathrm{G}_{2}\right)-\mathrm{n}_{2}^{4} \mathrm{~F}\left(\mathrm{G}_{1}\right)-\mathrm{n}_{1} \mathrm{~F}\left(\mathrm{G}_{2}\right)$

$+4 \mathrm{~m}_{1} \mathrm{n}_{2}^{2}\left(\mathrm{~m}_{1} \mathrm{n}^{2}{ }_{2}+2 \mathrm{~m}_{2} \mathrm{n}_{1}\right)+4 \mathrm{~m}_{2}^{2} \mathrm{n}^{2}{ }_{1}$

$+8 \mathrm{~m}_{1} \mathrm{~m}_{2}\left(\mathrm{n}_{1} \mathrm{n}_{2}^{2}-2 \mathrm{n}_{2}-\mathrm{m}_{2}\right)$.

The proof of the following theorem follows by applying Theorem 2.3 of [2] and Theorem 8, respectively.

Theorem 9. Let $\mathrm{G}_{\mathrm{i}}$ be a graph with $\mathrm{n}_{\mathrm{i}}$ vertex and $\mathrm{m}_{\mathrm{i}}$ edges, $\mathrm{i}=1,2$. Then $\overline{H M}_{1}^{*}\left(\mathrm{G}_{1}\left[\mathrm{G}_{2}\right]\right)=\mathrm{n}_{2}^{4} \mathrm{HM}\left(\mathrm{G}_{1}\right)+\mathrm{n}_{1} \mathrm{HM}\left(\mathrm{G}_{2}\right)$

$+\mathrm{n}_{2}^{2} \mathrm{M}_{1}\left(\mathrm{G}_{1}\right)\left(\mathrm{n}_{2} \mathrm{n}_{1}-2\right)+\mathrm{M}_{1}\left(\mathrm{G}_{2}\right) \mathrm{n}_{1}\left(\mathrm{n}_{1} \mathrm{n}_{2}-2\right)$

$-\mathrm{n}_{2}^{4} \mathrm{M}_{2}\left(\mathrm{G}_{1}\right)-\mathrm{n}_{1} \mathrm{M}_{2}\left(\mathrm{G}_{2}\right)-\mathrm{n}_{2}{ }_{2} \mathrm{~F}\left(\mathrm{G}_{1}\right)-\mathrm{n}_{1} \mathrm{~F}\left(\mathrm{G}_{2}\right)$

$+4 \mathrm{~m}_{1} \mathrm{n}_{2}^{2}\left(\mathrm{~m}_{1} \mathrm{n}^{2}{ }_{2}+2 \mathrm{~m}_{2} \mathrm{n}_{1}\right)+4 \mathrm{~m}_{2}^{2} \mathrm{n}^{2}$

$+8 \mathrm{~m}_{1} \mathrm{~m}_{2} \mathrm{n}_{2}\left(\mathrm{n}_{1} \mathrm{n}_{2}-2\right)$.

Using Theorem 9, we have the following examples.

Example 2.11. The vertex hyper-zagreb indices of $\mathrm{P}_{n}\left[\mathrm{P}_{m}\right]$ and $\mathrm{C}_{\mathrm{n}}\left[\mathrm{C}_{\mathrm{m}}\right]$ are given by

(i) $\overline{H M}^{*}\left(\mathrm{P}_{\mathrm{n}}\left[\mathrm{P}_{\mathrm{m}}\right]\right)=4 \mathrm{~m}^{4}\left(\mathrm{n}^{2}-\mathrm{n}-1\right)$

$+m^{3}\left(20 n^{2}+22 n\right)-4 m^{2}\left(3 m^{2}+n-7\right)+4 n(n-1)-16 m+20 m n$.

(ii) $\overline{H M}_{1}^{*}\left(\mathrm{C}_{\mathrm{n}}\left[\mathrm{C}_{\mathrm{m}}\right]\right)=4 \mathrm{~m}^{4}\left(\mathrm{n}^{2}-\mathrm{n}+1\right)$

$+4 m^{3}\left(5 n^{2}-4 n\right)-8 m^{2}\left(n^{2}+n-16\right)$

$-4 n^{2}(2 m-1)+4 m(3 n-4)$.

Example 2.12. The open fence graph is defined as $\mathrm{P}_{n}\left[\mathrm{P}_{2}\right]$. Similarly, the closed fence graph is defined as $\mathrm{C}_{\mathrm{n}}\left[\mathrm{P}_{2}\right]$.

The vertex hyper-Zagreb indices of the open and closed fence graphs are given by

(i) $\overline{H M}_{1}^{*}\left(\mathrm{P}_{\mathrm{n}}\left[\mathrm{P}_{2}\right]\right)=228 \mathrm{n}^{2}+132 \mathrm{n}-168$.

(ii) $\overline{H M}^{*}\left(\mathrm{C}_{\mathrm{n}}\left[\mathrm{P}_{2}\right]\right)=168 \mathrm{n}^{2}+17 \mathrm{n}$.

\section{References}

[1] Ashrafi A.R., Doslic T. and A. Hamzeh, The Zagreb coindices of graph operations, Discrete Appl. Math.158,(2010) ,1571-1578.

[2] Basavanagoud B. and S. Patila, note on hyper-Zagreb index of graph operations, Iran. J.Math. Chem. 7, (2016), 89-92.

[3] Bollobs B.,and P.Erds, Graphs of extremal weights. Ars Combinato ria, 50, (1998) 225-233.

[4] Doslic T., Vertex -weighted wiener polynomials for composite graphs, Ars Math. Contemp.1 (2008), 66-80.

[5] Fath-Tabar G.H, Old and new Zagreb indices of graphs, MA TCH Commun.Math.Comput.Chem. 65, (2011), 79-84

[6] Furtula B. and I. Gutman, A forgotten topological index, J. Math. Chem. 53, (2015), 1184-1190.

[7] Gao W., Jamil M.K. and M. R. Farahani, The hyper-Zagreb index and some graphoperations, J. Appl. Math. Comput. 54(1-2), (2015), 263-275

[8] Gutman I., Degree-based topological indices, Croat.Chem. acta 86, (2013), 351-361.

[9] Gutman I., On the origin of two degree -based topological indices, Bull.Acad. Serbe Sct.Arts (Cl.Sci.Math.Natur) 146 , (2014 ), 39-52.

[10] Gutman I., Das K. C, The first Zagreb index 30 years after, MATCH Commun. Math. Comput. Chem. 50, (2004), 83-92.

[11] Gutman I., Furtula B., Kovijanic Vukicevic Z. and G.popivoda, Zagreb indices and coindices, MATCH Commun. Math. Comput. Chem. 74, (2015), 5-16.

[12] Gutman I., Tomovice Z., On the application of line graphs in quantitative structure-property studies, J. Serb.Chem. soc. 65, (2000), 577-580.

[13] Gutman I. and N. Trinajstic, Graph theory and molecular orbitals. Total $\psi$-electron energy of alternant hydrocarbons. Chemical physics Letters, 17(4), (1972), 535-538.

[14] Khalifeh M.H, Yousefi-Azari H. and A.R. Ashrafi, The first and second Zagreb indices of some graph operations, Discrete Appl. Math. 157, (2009), 804-811.
[15] Pattabiraman K. and M. Seenivasan, Bounds on Vertex Zegrab Indices of Graphs, Global Journal of Frontier Science Research (GJFSR), Volume XVII, IssuseVI,Version1, ( 2017), 43-47.

[16] Pattabiraman K. and M. Vijayaragavan, Hyper Zagreb indices and its coindices of graphs, Bull. Int. Math. virtual Institute 7,(2017), 31-41.

[17] Randic M., Characterization of molecular branching. Journal of the American Chamical Society ,97(23), (1975), 6609-6615.

[18] Shirdel G.H., Rezapour H. and A.M. Sayadi, The hyper- Zagreb index of graph operations, Iranian J. Math. Chem.4,(2013),213220.

[19] Tavakoli M., Rahbarnia F. and A.R. Ashrafi, Eccentric connectivity and Zagreb coundices of the generalized hierarchical product of graphs, J. Discrete Mathematics . DOI 10.1155/2014/292679 (2014), 5 pages.

[20] Todeschini R. and V. Consonni, Handbook of molecular descritor (vol.11).John Wiley\&sons, (2008).

[21] Veylaki M., Nikmehr M.J. and H.A. Tavallae, The third and hyper Zagreb coindices of some graph operations, J. Appl.Math. Comput. 50, (2016), 315-325. 